\title{
A Semi-Phenomenological Model for the Material Gain of Broadband MQW-SOAs
}

\author{
T. Motaweh, P. Morel, A. Sharaiha, M. Guégan \\ ENIB, Lab-STICC UMR CNRS (6285) \\ CS 73862, 29238 Brest Cedex 3, France \\ motaweh@enib.fr
}

\begin{abstract}
We propose a semi-phenomenological model to calculate the material gain of multiple quantum well broadband Semiconductor Optical Amplifier (SOA). We show that this proposed model permits to fit well the experimental results of a broadband SOA gain and noise figure over a range of $100 \mathrm{~nm}$.
\end{abstract}

Keywords-Material gain, Modeling, MQW-SOA, Broadband optical bandwidth

\section{INTRODUCTION}

New broadband SOAs based on Multiple Quantum Well (MQW) structures have been developed for metropolitan and access networks as for example in the framework of the French ANR AROME and UltraWIDE projects [1]. Such new devices need to be simply though accurately modeled to be used in optical systems design tools. In fact, a good knowledge of their material gain over a broad range of carrier densities and wavelengths is required. However, using detailed physical models in simulators at the system level is cumbersome and could be not always valid in all operating conditions. For bulk structures, an analytical model has already been proposed [2] where the gain spectrum behavior is approximated by a polynomial model. But for QW structures, it's not easy to use this type of approximation because the shape of the gain spectrum is completely modified as the carrier density changes. In this work, we introduce in section II a novel semiphenomenological model to calculate the material gain of a MQW-SOA. We also show that this model can be easily adapted to determine the spontaneous emission. In section III, we present the comparison between simulation and experimental results of a broadband SOA gain and noise figure over a range of $100 \mathrm{~nm}$.

\section{MODEL DEFINITION}

The proposed model is based on a semi-phenomenological approach where the physical parameters in the equations describing a single QW in [3-4] are considered only as mathematical elements. We have developed our material gain expression by taking into account a system of two optical transitions with only two discrete levels in the conduction band and two discrete levels in the valence band, which is:

$$
\begin{array}{r}
g_{m}(N, \lambda)=g_{0} \frac{\lambda}{d} \sum_{\mathrm{n}=0}^{1}\left(1+\frac{E_{c, \mathrm{n}}}{\varepsilon_{c, \mathrm{n}}(\lambda)}\right)\left(f_{c, \mathrm{n}}(N, \lambda)-\right. \\
\left.f_{v, \mathrm{n}}(N, \lambda)\right)\left(\frac{1}{2}+\frac{1}{\pi} \operatorname{atan}\left(2 \pi \tau_{i n} c_{0}\left(\frac{1}{\lambda}-\frac{1}{\lambda_{c v, \mathrm{n}}}\right)\right)\right)
\end{array}
$$

where $\mathrm{n}$ indicates the optical transition; $c_{0}$ is the speed of light in vacuum and $h$ the Planck's constant; $d$ corresponds to the well thickness; $\tau_{\text {in }}$ is the intraband relaxation time; $g_{0}$ is a coefficient which depends on the composition of the material and the used structure: it is fixed to $2757 \mathrm{~m}^{-1}$ in our case. The material gain coefficient $\left(g_{m}{ }^{\prime}(N, \lambda)\right)$, which is used to calculate the spontaneous emission of SOAs, can be simply defined from (1) by classically replacing $\left(f_{c, \mathrm{n}}-f_{v, \mathrm{n}}\right)$ by $f_{c, \mathrm{n}}\left(1-f_{v, \mathrm{n}}\right)$. The Fermi functions $f_{c, \mathrm{n}}(N, \lambda)$ and $f_{v, \mathrm{n}}(N, \lambda)$ are given by:

$$
f_{\mathrm{x}, \mathrm{n}}(N, \lambda)=\left[1+\exp \left(\frac{\varepsilon_{\mathrm{x}, \mathrm{n}}(\lambda)-E_{\mathrm{f}, \mathrm{x}}(N)}{k_{B} T}\right)\right]^{-1}
$$

Where $\mathrm{x}$ denotes $c$ or $v$ index, $T$ is the absolute temperature, $k_{B}$ is the Boltzmann constant and $E_{\mathrm{f}, \mathrm{x}}(N)$ is the quasi Fermi level. Thanks to our simplifying assumptions, $E_{\mathrm{f}, \mathrm{X}}(N)$ can be calculated analytically as the solution of a $2^{\text {nd }}$ order equation:

$E_{f, X}(N)= \pm k_{B} T \log \left(\frac{-A_{X}+\sqrt{A_{x}{ }^{2}-4\left(1-e^{\frac{N}{N_{X}}}\right) e^{-B_{X}}}}{2 e^{-B_{X}}}\right)$

Where $A_{\mathrm{x}}=\sum_{\mathrm{n}=0}^{1} e^{-\frac{E_{\mathrm{x}, \mathrm{n}}}{k_{B} T}}, B_{\mathrm{x}}=\sum_{\mathrm{n}=0}^{1} \frac{E_{\mathrm{x}, \mathrm{n}}}{k_{B} T}$ and $N_{\mathrm{x}}=\frac{4 \pi k_{B} T m_{x}}{d h^{2}}$. \pm denotes that $\mathrm{E}_{\mathrm{f}, \mathrm{c}}(\mathrm{N})$ is positive and $\mathrm{E}_{\mathrm{f}, \mathrm{v}}(\mathrm{N})$ negative.

We also define the energy levels in the conduction band $\varepsilon_{c, \mathrm{n}}(\lambda)$ and in the valence band $\varepsilon_{v, \mathrm{n}}(\lambda)$ as:

$$
\begin{aligned}
& \varepsilon_{c, \mathrm{n}}(\lambda)=E_{c, \mathrm{n}}+\frac{h c}{\left(1+\frac{m_{c}}{m_{v}}\right)}\left(\frac{1}{\lambda}-\frac{1}{\lambda_{c v, \mathrm{n}}}\right) \\
& \varepsilon_{v, \mathrm{n}}(\lambda)=-E_{v, \mathrm{n}}-\frac{h c}{\left(1+\frac{m_{v}}{m_{c}}\right)}\left(\frac{1}{\lambda}-\frac{1}{\lambda_{c v, \mathrm{n}}}\right)
\end{aligned}
$$

Where $E_{c, \mathrm{n}}$ and $E_{v, \mathrm{n}}$ are given as $E_{c, 0}=E_{0}, E_{c, 1}=E_{0}+\Delta E$, $E_{v, 0}=\frac{h c_{0}}{\lambda_{c v, 0}}-E_{0}$ and $E_{v, 1}=\frac{h c_{0}}{\lambda_{c v, 1}}-\left(E_{0}+\Delta E\right)$.

In the preceding equations, $\lambda_{c v, 0}$ and $\lambda_{c v, 1}$ are the wavelengths of respectively the first and the second optical transition; $m_{c}$ and $m_{v}$ depend on the composition of the material and also of the structure: they are calculated in our case as $m_{c}=0.0482 m_{0}$ and $m_{v}=0.454 m_{0}$ where $m_{0}$ is the mass of the electron.

We highlight the fact that our material gain equation (1) needs a reduced set of parameters $\left(\lambda_{\mathrm{cv}, 0}, \lambda_{\mathrm{cv}, 1}, E_{0}, \Delta \mathrm{E}, m_{c}, m_{v}\right.$, $\tau_{\text {in }}$ and $\left.g_{0}\right)$. Some of them are fixed like $m_{c}, m_{v}$ and $g_{0}$. It shall also be noticed that no additional parameter is required to calculate the spontaneous emission. 


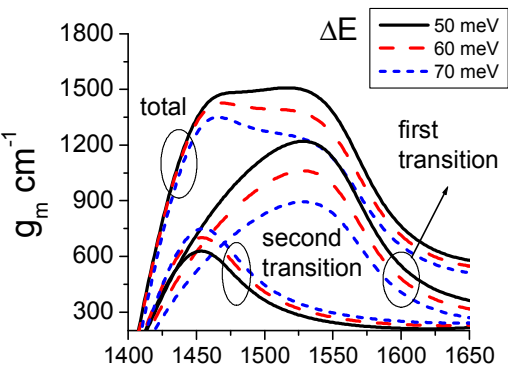

a)

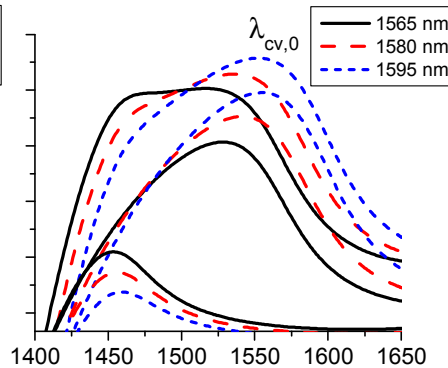

b)

Wavelength [nm]

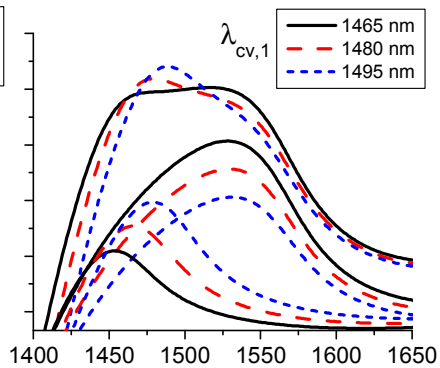

c)

Fig. 1. Material gain spectrum of the first and second optical transition and of the total material gain for different values of $\Delta \mathbf{E}$ (a), $\boldsymbol{\lambda}_{\mathbf{c v}, \mathbf{0}}$ (b) and $\boldsymbol{\lambda}_{\mathbf{c v}, \mathbf{1}}$ (c) for a given carrier density $\mathrm{N}=2.510^{24} \mathrm{~m}^{-3}$.

Fig. 1 shows the influence of some of the unfixed parameters $\left(\Delta \mathrm{E}, \lambda_{\mathrm{cv}, 0}, \lambda_{\mathrm{cv}, 1}\right)$ to represent their influence on the shape of the material gain spectrum, at a given carrier density of $2.510^{24} \mathrm{~m}^{-3}$. It can be clearly seen in Fig. 1a that $\Delta \mathrm{E}$ modifies the gain value of higher wavelengths and operates a redistribution of the gain between the two transitions. Concerning the $\lambda_{c v, 0}$ and $\lambda_{c v, 1}$ parameters, their increase changes, of course, the spectrum gain response of their corresponding transition $\left(\lambda_{c v, 0} \leftrightarrow\right.$ first transition, $\lambda_{c v, 1} \leftrightarrow$ second transition) by shifting its towards higher wavelengths and enhancing its gain. However, as we can remark in Fig. 1b and 1c, it induces less gain to the transition for which the wavelength stays constant. Therefore, we can obtain the appropriate gain spectral shape by adjusting these parameters.

\section{EXPERIMENTAL VALIDATION}

In order to validate the material gain model described in the previous section, we used the gain and Noise Figure (NF) characterization results of a broadband MQW-SOA from Alcatel III-V Lab. $g_{m}(N, \lambda)$ and $g_{m}{ }^{\prime}(N, \lambda)$ equations were implemented into our SOA simulation tool [5]. The optimal model parameter values $\left(\mathrm{E}_{\mathrm{c}, 0}=106 \mathrm{meV}, \Delta \mathrm{E}=72.5 \mathrm{meV}\right.$, $\lambda_{\mathrm{cv}, 0}=1.593 \mathrm{~nm}, \lambda_{\mathrm{cv}, 1}=1.469 \mathrm{~nm}$ and $\tau_{\mathrm{in}}=26.7 \mathrm{fs}$ ) were obtained after an automatized optimization procedure.

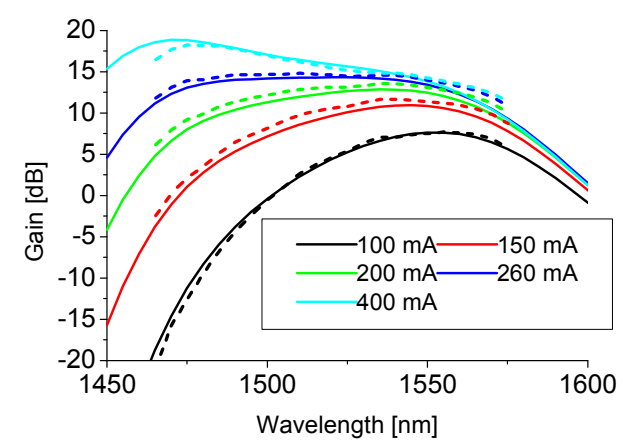

Fig. 2. SOA gain spectrum for five bias currents. Lines are theoretical results, dashes are experimental results.

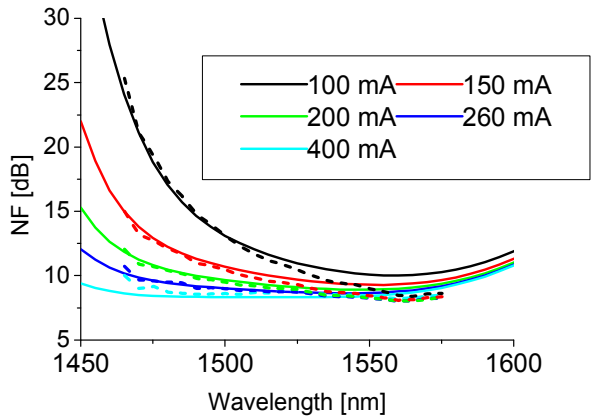

Fig. 3. SOA NF spectrum for five bias currents. Lines are theoretical results, dashes are experimental results.

Fig. 2 and 3 show the SOA gain and NF spectra for several biasing currents. It can be seen that the model permits to reproduce with a good concordance the spectral shape of the MQW SOA for a wide range of bias currents.

\section{CONCLUSION}

We have presented and validated a semi-phenomenological model devoted to calculate the material gain of MQW-SOAs. We showed that this model permits to reproduce accurately the experimental results of a broadband SOA gain and noise figure over a wide range of wavelengths and bias currents.

\section{ACKNOWLEDGMENT}

This work is supported by the French ANR research agency in the frame of UltraWIDE project ANR 2010 VERS 01106.

\section{REFERENCES}

[1] Carrère $\mathrm{H}$. et al, "Large optical bandwidth and polarization insensitive semiconductor optical amplifiers using strained InGaAsP quantum wells," Applied Physics Letters, vol.97, 2010.

[2] Leuthold J. et al, "Material gain of bulk $1.55 \mu \mathrm{m}$ InGaAsP/InP semiconductor optical amplifiers approximated by a polynomial model," J. of Applied Physics, vol.87, no.1, pp.618-620, 2000.

[3] Asada M. et al, "Gain and intervalence band absorption in quantum-well lasers," IEEE J. of Quantum Electronics, vol.20, no.7, pp.745-753, 1984.

[4] Makino T., "Analytical formulas for the optical gain of quantum wells," IEEE J. of Quantum Electronics, vol.32, no.3, pp.493,501, 1996.

[5] Morel P., Sharaiha A., "Wideband Time-Domain Transfer Matrix Model Equivalent Circuit for Short Pulse Propagation in Semiconductor Optical Amplifiers," IEEE J. of Quantum Electronics, vol.45, no.2, pp.103-116, 2009. 